\title{
Ultrassonografia testicular em bovinos jovens da raça Nelore criados em sistema extensivo
}

[Testicular ultrasonography in young Nelore bulls raised in extensive management system]

\author{
D.J. Cardilli ${ }^{1}$, G.H. Toniollo ${ }^{1}$, A.A. Pastore ${ }^{2}$, J.C. Canola $^{2}$, J.A. Oliveira $^{2}$, M.E.Z. Mercadante ${ }^{3}$
}

\author{
${ }^{1}$ FCAV-UNESP-Jaboticabal, SP \\ ${ }^{2}$ Médico veterinário autônomo \\ ${ }^{3}$ Estação Experimental de Zootecnia - Sertãozinho, SP
}

\begin{abstract}
RESUMO
Este estudo visou avaliar o padrão ultrassonográfico do parênquima testicular de touros jovens da raça Nelore, desde a fase peripuberal até a puberdade, estabelecer padrões fisiológicos e também verificar se existe diferença de ecogenicidade entre animais púberes e pré-púberes na mesma idade. Foram realizados exames ecográficos dos testículos de 19 bovinos aos 10,12, 14, 16 e 18 meses de idade. O padrão ultrassonográfico do parênquima testicular mostrou-se homogêneo e com ecogenicidade moderada. A ecogenicidade testicular aumentou em proporção direta com a idade dos animais. Não houve diferença significativa entre a ecogenicidade testicular de animais púberes e pré-púberes na mesma idade.
\end{abstract}

Palavras-chave: trato reprodutor masculino, ecografia, bovino

\begin{abstract}
The aim of this study was to evaluate the testicular parenchyma of young Nelore bulls through standard ultrasound, in order to establish the physiological standards, as well as differences in echogenicity between pre-pubescent and pubescent animals of the same age. Testis of 19 young Nelore bulls were evaluated by ultrasonography at 10,12,14, 16 and 18 months old. Homogeneous and moderate testicular parenchyma echogenicity was observed in all animals. Testicular echogenicity increased with the age of the animal. There were no significant differences in testicular echogenicity between pre-pubescent and pubescent animals with the same age.
\end{abstract}

Keywords: male reproductive tract, echography, bovine

\section{INTRODUÇÃO}

O exame ultrassonográfico dos testículos é um método não invasivo e rápido, que, aliado aos dados de exame clínico, pode conduzir ao diagnóstico precoce de desordens desse órgão (Pechman e Eilts, 1987). No entanto, a análise da imagem é subjetiva, pois depende da experiência do examinador. Sendo assim, instituir valores aos diferentes tons da escala de cinza possibilita a determinação quantitativa do padrão de normalidade para a ecogenicidade do parênquima testicular.

Recebido em 28 de outubro de 2011

Aceito em 24 de novembro de 2011

E-mail: djcardilli@yahoo.com.br
Pechman e Eilts (1987) e Abdel-Razek e Ali (2005) identificaram as estruturas anatômicas do trato reprodutivo de touros adultos Bos Taurus por meio do exame ultrassonográfico, e relataram que o parênquima testicular é homogêneo e moderadamente ecogênico e que o mediastino testicular é uma estrutura linear de aproximadamente $5 \mathrm{~mm}$ de largura, hiperecoica em relação ao parênquima testicular.

Alguns autores estudaram a ecogenicidade do parênquima testicular em touros desde a fase peripuberal até a puberdade, e descreveram que esta aumenta com o decorrer da idade do animal. 
Observaram também que o parênquima testicular de touros que atingem a puberdade mais precocemente dentro de um rebanho é hiperecoico quando comparado àqueles mais tardios (Chandolia et al., 1997; Aravindakshan et al., 2000; Kastelic et al., 2001; Brito et al., 2002; Brito et al., 2004 e Abdel-razek e Ali, 2005).

Desordens como tumores e processos inflamatórios são tipicamente hipoecoicos em contraste com a ecogenicidade moderada de testículos maturos (Horstman et al., 1994 e Simon et al., 2001); sendo assim, a identificação de tais desordens em animais pré-púberes, os quais são o foco deste estudo, é mais difícil devido à baixa ecogenicidade testicular nesta fase. Tais afirmações demonstram ainda mais a importância de se descrever o padrão ultrassonográfico normal do parênquima testicular de bovinos pré-púberes.

Os objetivos deste estudo foram avaliar, por meio de programa de análise de imagem, retirando-se a subjetividade, o padrão ultrassonográfico do parênquima testicular de touros jovens da raça Nelore desde a fase peripuberal até a puberdade, estabelecer padrões fisiológicos, e também verificar se existe diferença de ecogenicidade entre animais púberes e pré-púberes na mesma idade, para, desta maneira, apresentar uma importante contribuição para o exame andrológico de touros.

\section{MATERIAL E MÉTODOS}

Foram realizados exames ultrassonográficos dos testículos de 19 bovinos hígidos da raça Nelore, a cada 60 dias, dos 10 aos 18 meses de idade. O rebanho, criado em sistema extensivo com suplementação de sal mineralizado, estava localizado na cidade de Guararapes-SP, Brasil, latitude $-21^{\circ} 15^{\prime} 39^{\prime \prime}$ e longitude $50^{\circ} 38^{\prime} 34^{\prime \prime}$.

Os exames ultrassonográficos foram realizados com aparelho Pie Medical Scanner 200C, ligado a um transdutor linear de $8 \mathrm{MHz}$. Após contensão dos animais em tronco e aplicação de gel acústico diretamente sobre o escroto, foram realizadas varreduras em planos transversais e longitudinais nos testículos direito e esquerdo. Ao início de cada dia de trabalho o aparelho de ultrassonografia foi calibrado sempre de uma mesma maneira quanto à intensidade de brilho e também quanto à posição do foco principal.
As imagens selecionadas foram transferidas diretamente ao computador por meio do software Eview-Echo Image Viewer, versão 1.00, Pie Medical Equipament B.V., copyright Pie Medical (EIV). Com o auxílio do software EIV, foram selecionadas duas regiões de interesse (RI) em cada plano de varredura nos testículos direito e esquerdo. Cada RI foi delimitada por um quadrado de $6,3 \mathrm{~mm}$ de lado, e elas foram localizadas lateral e medialmente ao mediastino testicular no plano transversal, e no polo ventral e dorsal do testículo, excluindo-se o mediastino testicular, no plano longitudinal. As RI contiveram o máximo de parênquima testicular, sem englobar pele, túnicas, epidídimo e o mediastino testicular.

Automaticamente o software EIV calculou a ecogenicidade do parênquima testicular nas RI, em uma escala que variou de zero a $100 \%$, sendo $0 \%$ para uma imagem totalmente preta ao monitor (parênquima testicular menos ecogênico), e $100 \%$ para uma imagem totalmente branca (parênquima testicular mais ecogênico). Também por meio do software EIV foram feitas as aferições da espessura do mediastino testicular, sempre no plano longitudinal de varredura dos testículos.

Os exames andrológicos foram feitos segundo a metodologia descrita por Krause (1993). A mensuração da circunferência escrotal (CE) foi realizada com uma fita milimetrada; para esta característica, adotou-se como unidade o centímetro $(\mathrm{cm})$. Para se obter o volume testicular (VT), foi usada a fórmula idealizada por Unaniam et al. (2000): Vol $=2\left\{(\mathrm{r} / 2)^{2} \mathrm{x} \pi \mathrm{x}\right.$ $\mathrm{h}\}$, onde $\mathrm{r}=$ largura $/ 2 ; \mathrm{h}=$ comprimento (considerando-se os testículos e excluindo-se a cauda do epidídimo) e $\pi=3,14$, sendo o valor obtido expresso em centímetros cúbicos $\left(\mathrm{cm}^{3}\right)$. O paquímetro foi utilizado para fornecer $\mathrm{o}$ comprimento e a largura de cada testículo. Padronizou-se que os valores aferidos no testículo esquerdo foram aqueles utilizados na fórmula e as aferições foram efetuadas sempre pelo mesmo indivíduo.

A colheita do sêmen foi realizada por eletroejaculação aos 14, 16 e 18 meses de idade, sempre após os exames ultrassonográficos, sendo que os parâmetros avaliados e a metodologia de análise do sêmen foram os mesmos utilizados por Brito et al. (2004). 
O parâmetro estabelecido para a puberdade neste estudo foi: ejaculado com mínimo de $50 \times 10^{6}$ espermatozoides, pelo menos $10 \%$ de motilidade progressiva e $70 \%$ ou mais de células normais no ejaculado, semelhante ao utilizado por Brito et al. (2004).

A ecogenicidade do parênquima testicular foi analisada pelo método dos quadrados mínimos, por meio do procedimento General Linear Model (GLM) do programa SAS (SAS 9.1, SAS Institute, Cary, NC, USA), utilizando-se um modelo que incluiu os efeitos de touro (1-19), planos (longitudinal e transversal), testículos (direito e esquerdo), idade e interações entre esses efeitos. Quando o efeito ou a interação foi significativa pelo teste $\mathrm{F}$, as médias foram comparadas pelo teste de Tukey a 5\% de probabilidade. Foram feitas também análises de regressão das variáveis ecogenicidade do parênquima testicular, CE, VT e espessura do mediastino em função do tempo (idade em meses), e também correlações simples de Pearson entre as variáveis PE, VT e espessura do mediastino com a ecogenicidade do parênquima testicular.

\section{RESULTADOS}

O padrão ultrassonográfico do parênquima testicular de bovinos jovens da raça Nelore mostrou-se homogêneo e com ecogenicidade moderada (Fig. 1). Não houve diferença $(\mathrm{P}>0,05)$ entre as ecogenicidades dos testículos direito e esquerdo, sendo que as médias no período dos 10 aos 18 meses, foram, respectivamente, 29,55 e 29,19\% (Tab. 1). Por outro lado, entre os planos de varredura, foi observado que as imagens em plano transversal apresentaram maior ecogenicidade $(\mathrm{P}<0,05)$ que aquelas em plano longitudinal, cujas médias dos 10 aos 18 meses foram, respectivamente, 30,80 e $27,84 \%$ (Tab. 2).

As médias da ecogenicidade do parênquima testicular aos 10, 12, 14, 16 e 18 meses de idade foram, respectivamente, 18,$47 ; 28,48 ; 25,19$; 29,53 e $45,17 \%$ (Tab. 3), ou seja, aumentou no intervalo de 10 a 12 meses e, posteriormente, houve uma pequena, porém significativa, diminuição. Depois, no intervalo de 14 a 18 meses, foi encontrado outro aumento. Apenas os valores obtidos aos 16 e aos 12 meses não diferiram entre si $(\mathrm{P}>0,05)$.

A análise de regressão da ecogenicidade (variável dependente) em função da idade do animal em meses (variável independente) foi significativa $(\mathrm{P}<0,05)$, sendo que a de terceiro grau foi a que melhor representou os dados observados. A equação observada foi: $\mathrm{y}=-11,8$ $+45,6 x-17,1 x^{2}+2,0 x^{3}$, em que $R^{2}=0,64$.

Tabela 1. Ecogenicidade do parênquima dos testículos direito e esquerdo (média \pm desvio-padrão), de bovinos jovens da raça Nelore, nos diferentes momentos de avaliação. UNESP - Jaboticabal, SP, 2011

\begin{tabular}{ccc}
\hline Idade (meses) & Testículo direito & Testículo esquerdo \\
\hline 10 & $18,60(76)^{*} \pm 4,70$ & $18,36(76) * \pm 3,35$ \\
12 & $28,50(76) \pm 6,76$ & $28,47(76) \pm 5,60$ \\
14 & $27,24(76) \pm 7,37$ & $23,25(76) \pm 5,82$ \\
16 & $28,26(76) \pm 7,14$ & $30,80(76) \pm 7,84$ \\
18 & $45,15(76) \pm 5,35$ & $45,18(76) \pm 5,38$ \\
\hline $10-18$ & $29,55 \mathrm{a}(380)^{*} \pm 10,83$ & $29,19 \mathrm{a}(380)^{*} \pm 10,84$ \\
\hline
\end{tabular}

Médias seguidas de letras diferentes nas linhas diferem entre si pelo teste Tukey $(\mathrm{p}<0,05)$. * Número de observações.

Tabela 2. Ecogenicidade do parênquima testicular nos planos de varredura transversal e longitudinal (média desvio-padrão), de bovinos jovens da raça Nelore, nos diferentes momentos de avaliação (UNESP - Jaboticabal, SP, 2011)

\begin{tabular}{ccc}
\hline Idade (meses) & Transversal & Longitudinal \\
\hline 10 & $18,85(76)^{*} \pm 4,55$ & $18,09(76)^{*} \pm 3,50$ \\
12 & $28,14(76) \pm 6,61$ & $28,81(76) \pm 5,76$ \\
14 & $26,14(76) \pm 8,02$ & $24,24(76) \pm 5,52$ \\
16 & $32,64(76) \pm 8,93$ & $26,42(76) \pm 4,03$ \\
18 & $48,22(76) \pm 6,74$ & $42,11(76) \pm 4,65$ \\
$10-18$ & $30,80 \mathrm{a}(380)^{*} \pm 12,09$ & $27,84 \mathrm{~b}(380)^{*} \pm 9,25$ \\
\hline
\end{tabular}

Médias seguidas de letras diferentes nas linhas diferem entre si pelo teste Tukey $(\mathrm{p}<0,05) . *$ Número de observações. 


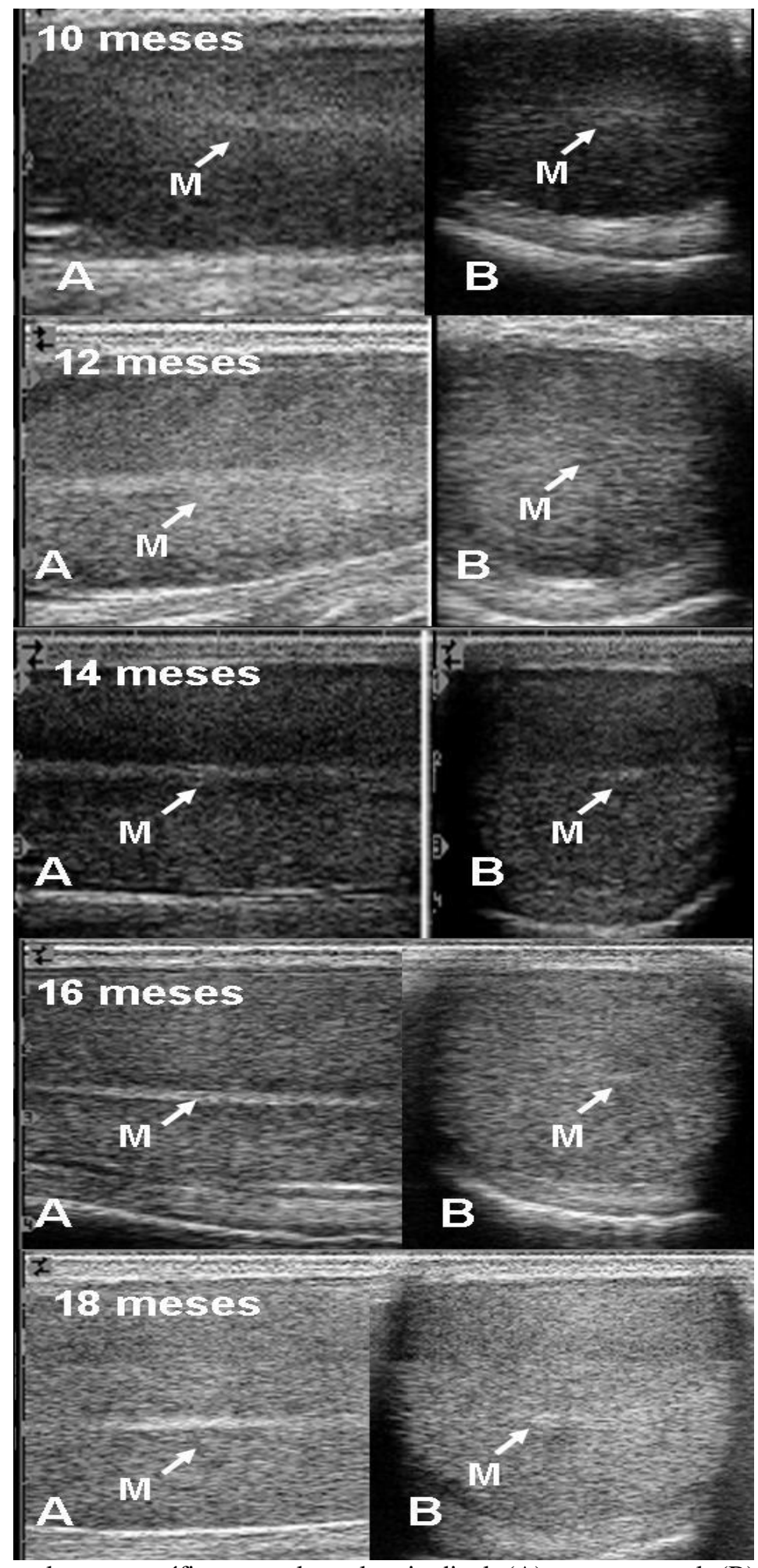

Figura 1. Imagem ultrassonográfica em plano longitudinal (A) e transversal (B), demonstrando o parênquima testicular normal e o mediastino (M) de bovinos jovens da raça Nelore, aos 10, 12, 14, 16 e 18 meses de idade. Note o padrão ultrassonográfico homogêneo e o aumento da ecogenicidade com o decorrer da idade (UNESP - Jaboticabal, SP, 2011). 
Ultrassonografia testicular...

Tabela 3. Ecogenicidade do parênquima testicular, espessura do mediastino (Emed), circunferência escrotal (CE) e volume testicular (VT) (média \pm desvio-padrão), de bovinos jovens da raça Nelore, em cada momento de avaliação (UNESP - Jaboticabal, SP, 2011)

\begin{tabular}{ccccc}
\hline Idade (meses) & Ecogenicidade $(\%)$ & Emed(mm) & $\mathrm{CE}(\mathrm{cm})$ & $\mathrm{VT}\left(\mathrm{cm}^{3}\right)$ \\
\hline 10 & $18,47 \mathrm{a} \pm 4,07$ & $2,20 \mathrm{a} \pm 0,31$ & $18,28 \mathrm{a} \pm 1,34$ & $78,77 \mathrm{a} \pm 24,65$ \\
12 & $28,48 \mathrm{~b} \pm 6,19$ & $2,28 \mathrm{a} \pm 0,34$ & $19,37 \mathrm{a} \pm 1,51$ & $102,76 \mathrm{a} \pm 34,24$ \\
14 & $25,19 \mathrm{c} \pm 6,93$ & $2,40 \mathrm{a} \pm 0,35$ & $21,68 \mathrm{~b} \pm 1,94$ & $168,73 \mathrm{~b} \pm 45,83$ \\
16 & $29,53 \mathrm{~b} \pm 7,58$ & $2,33 \mathrm{a} \pm 0,31$ & $23,10 \mathrm{~b} \pm 2,11$ & $199,28 \mathrm{~b} \pm 54,52$ \\
18 & $45,17 \mathrm{~d} \pm 6,53$ & $2,58 \mathrm{~b} \pm 0,37$ & $26,21 \mathrm{c} \pm 1,59$ & $356,07 \mathrm{c} \pm 83,89$ \\
\hline
\end{tabular}

Médias seguidas de letras diferentes nas colunas diferem entre si pelo teste Tukey $(\mathrm{p}<0,05)$. $\mathrm{O}$ número de observações para cada média obtida na variável ecogenicidade é de 152, enquanto para as demais variáveis, Emed, PE, é de 19.

O mediastino testicular apresentou-se, em plano longitudinal, como uma linha hiperecoica no centro do parênquima testicular, com espessura variando entre $1,7 \mathrm{~mm}$ e $3,3 \mathrm{~mm}$, ao passo que, em plano transversal, como um ponto hiperecoico no centro do parênquima testicular. Tal estrutura foi mais facilmente identificada e mostrou-se mais ecogênica nos animais mais velhos, e sua espessura aumentou em proporção direta com a idade dos animais, sendo que as médias obtidas aos $10,12,14,16$ e 18 meses foram, respectivamente, 2,20;2,28;2,40;2,33 e 2,58 milímetros (Tab. 3). A regressão linear da espessura do mediastino testicular (variável dependente) em função da idade em meses (variável independente) foi significativa $(\mathrm{P}<0,05)$, e a seguinte equação do primeiro grau foi obtida: $\mathrm{y}=1,89+0,04 \mathrm{x}$, em que $\mathrm{R}^{2}=0,31$.

A CE e o VT também aumentaram à medida que os animais foram ficando mais velhos (Tab. 3); a regressão linear de ambas as variáveis dependentes (CE e VT) em função da idade em meses (variável independente) foi significativa $(\mathrm{P}<0,05)$, e foram estimadas, respectivamente, as seguintes equações de primeiro grau: $\mathrm{y}=9,91+0,90 \mathrm{x}$, em que $\mathrm{R}^{2}=0,73 \mathrm{e}$, $y=-159,52+26,80 x$, em que $R^{2}=0,73$.

A ecogenicidade do parênquima testicular apresentou correlação significativa e positiva com CE, VT e espessura do mediastino (Tab. 4).

Dos 19 animais que fizeram parte deste estudo, apenas 32\% (6/19) apresentaram sêmen compatível com puberdade somente aos 18 meses de idade. A média da ecogenicidade do parênquima testicular para os animais púberes aos 18 meses foi de $44,10 \%$, enquanto a média dos animais pré-púberes na mesma idade foi de $45,66 \%$ (Tab. 5), sendo que estes valores não diferiram $(\mathrm{P}>0,05)$ entre si.

Tabela 4. Estimativas de correlações simples de Pearson entre a variável ecogenicidade do parênquima testicular e as variáveis circunferência escrotal (CE), volume testicular (VT) e espessura do mediastino (Emed), em bovinos jovens da raça Nelore (UNESP - Jaboticabal, SP, 2011)

\begin{tabular}{c|cccc}
\hline & Ecogenicidade & CE & VT & Emed \\
\hline \multirow{2}{*}{ Ecogenicidade } & 1 & 0,81 & 0,75 & 0,26 \\
$\mathrm{p}$ & & $<, 0001$ & $<, 0001$ & 0,0003 \\
\hline
\end{tabular}

$\mathrm{p}=$ probabilidade estatística $(\mathrm{p}<0,05)$.

Tabela 5. Ecogenicidade do parênquima testicular para os bovinos jovens da raça Nelore, púberes e prépúberes (média \pm desvio-padrão), nos diferentes momentos de avaliação (UNESP - Jaboticabal, SP, 2011)

\begin{tabular}{ccc}
\hline Idade $($ Meses $)$ & Púberes $(\%)$ & Pré-Púberes $(\%)$ \\
\hline 10 & $17,50 \mathrm{a}(48)^{*} \pm 3,03$ & $18,99 \mathrm{a}(104)^{*} \pm 4,41$ \\
12 & $27,44 \mathrm{a}(48)^{*} \pm 6,01$ & $28,96 \mathrm{a}(104)^{*} \pm 6,24$ \\
14 & $26,08 \mathrm{a}(48)^{*} \pm 7,72$ & $24,77 \mathrm{a}(104)^{*} \pm 6,53$ \\
16 & $29,83 \mathrm{a}(48)^{*} \pm 7,65$ & $29,39 \mathrm{a}(104)^{*} \pm 7,58$ \\
18 & $44,10 \mathrm{a}(48)^{*} \pm 6,47$ & $45,66 \mathrm{a}(104)^{*} \pm 6,53$ \\
\hline
\end{tabular}

Médias seguidas de letras diferentes nas linhas diferem entre si pelo teste Tukey $(\mathrm{p}<0,05)$. *O número de observações. 


\section{DISCUSSÃO}

O padrão ultrassonográfico do parênquima testicular de bovinos jovens da raça Nelore mostrou-se homogêneo e com ecogenicidade moderada, resultados semelhantes aos relatados por Pechman e Eilts (1987) e Abdel-Razek e Ali (2005), trabalhando com animais Bos taurus. Esse conhecimento é válido para comparação com estudos em que foram observadas alterações, como, por exemplo, em um trabalho também com bovinos jovens da raça Nelore, desenvolvido por Cardilli et al. (2009), no qual foram demonstradas alterações do padrão ultrassonográfico do parênquima testicular, as quais foram descritas como padrão heterogêneo, com múltiplos pontos hiperecoicos que não produziam sombra acústica.

Várias pesquisas demonstraram, por meio da ultrassonografia, o desenvolvimento dos testículos de touros jovens (Chandolia et al., 1997; Silva et al., 1997; Aravindakshan et al., 1999; Kastelic, 2001; Brito et al., 2002; Brito et al., 2004 e Abdel-razek e Ali, 2005). Os autores que trabalharam com touros Bos taurus relataram que a ecogenicidade do parênquima testicular aumentou com o decorrer da idade (Chandolia et al., 1997; Aravindakshan et al., 1999 e AbdelRazek e Ali, 2005), o que corrobora com os resultados obtidos no presente estudo, porém Silva et al. (1997), no único relato do gênero no qual foram utilizados apenas touros da raça Nelore, disseram que a ecogenicidade diminuiu com o passar da idade. Tal resultado foi explicado com base em possíveis diferenças de equipamento e metodologia de análise das imagens.

Vale ressaltar que a presente pesquisa apresenta um modelo inovador no armazenamento das imagens para posterior análise, pois, em todos os trabalhos citados anteriormente, as imagens eram gravadas em VHS e posteriormente digitalizadas, ao passo que neste estudo as imagens eram transferidas diretamente ao computador por meio do software EIV, o que pode minimizar perdas de qualidade nas imagens.

Chandolia et al. (1997) explicaram o aumento da ecogenicidade do parênquima testicular da seguinte maneira: primeiramente ocorre uma proliferação celular; após esta fase inicia-se a divisão das espermatogônias e formação de mais tipos de células maturas da espermatogênese; posteriormente acontece aumento da densidade das células, caracterizado pela formação das espermátides e consequente transformação destas em espermatozoides. Os mesmos autores relataram um período em que a ecogenicidade testicular diminuiu, exatamente como foi encontrado no presente estudo, e atribuíram este fato à formação do lúmen dos túbulos seminíferos e ao término da formação das células de Sertoli e consequente produção de fluido por estas células, necessários à espermatogênese.

O parênquima testicular nas imagens em plano transversal apresentou maior ecogenicidade que aquelas obtidas em plano longitudinal. Este resultado pode ser atribuído à influência do mediastino testicular, o qual não pode ser retirado das imagens em plano transversal, o que não acontece com as imagens em plano longitudinal, nas quais o parênquima testicular pode ser visualizado sem o mediastino. Hamm e Fobbe (1995), ao analisarem as imagens dos testículos de 143 meninos, mediram apenas a ecogenicidade testicular nas imagens obtidas em plano longitudinal, devido à presença do mediastino nas imagens em plano transversal. Chandolia et al. (1997) não mencionaram se houve ou não diferença significativa na ecogenicidade do parênquima testicular em imagens destes dois planos, entretanto Aravindakshan et al. (2000), ao estudarem o desenvolvimento testicular por ultrassonografia em 20 bovinos de origem taurina, relataram que não houve diferença significativa entre os planos transversal e longitudinal.

A espessura e a ecogenicidade do mediastino aumentaram com a idade dos animais. Tais resultados corroboram com os achados de AbdelRazek e Ali (2005), porém discordam dos resultados obtidos por Pechman e Eilts (1987), os quais ao trabalharem com animais Bos taurus, concluíram que a espessura do mediastino e sua ecogenicidade diminuíram com a idade. Segundo Sisson (1986), os túbulos seminíferos formam no mediastino uma rede, a rede do testículo (rete testis), resultando que, com o aumento da idade do animal, ocorrem importantes e consideráveis mudanças anatômicas nos túbulos seminíferos, os quais se tornam mais longos e "retorcidos" e aumentam em diâmetro, formando um lúmen (Hamm e Fobbe, 1995), o que pode explicar o aumento da espessura do mediastino testicular. 
Os resultados observados na presente pesquisa mostram que a ecogenicidade do parênquima testicular apresentou altas correlações positivas com CE e VT, ou seja, quando estas duas últimas variáveis aumentam, espera-se que a ecogenicidade também aumente. Dentre os autores que buscaram estabelecer a importância da ultrassonografia como indicador de potencial reprodutivo, Brito et al. (2004) relataram aumento da ecogenicidade testicular em animais da raça Canchim púberes quando comparados com pré-púberes, concluindo que a $\mathrm{CE}$, o peso e a idade são melhores indicadores de potencial reprodutivo do que a medida da ecogenicidade do parênquima testicular, pois apresentaram maiores valores em testes de sensibilidade e especificidade para esta característica.

Aravindakshan et al. (2000) também relataram que animais púberes apresentaram maior ecogenicidade testicular que os pré-púberes na mesma idade e sugeriram que a ultrassonagrafia pode ser utilizada para indicar touros com melhor potencial reprodutivo dentro de um rebanho. Ao contrário, no presente estudo, não foi observada diferença significativa $(\mathrm{P}>0,05)$ entre as ecogenicidades dos parênquimas testiculares de animais púberes e pré-púberes na mesma idade.

\section{CONCLUSÕES}

O padrão ultrassonográfico do parênquima testicular de touros jovens da raça Nelore é homogêneo, com ecogenicidade moderada, a qual aumenta com o decorrer da idade, assim como a espessura do mediastino testicular. A ecogenicidade do parênquima testicular apresenta alta correlação positiva com o volume testicular e a circunferência escrotal, não havendo diferença significativa entre as ecogenicidades dos parênquimas testiculares de touros púberes e pré-púberes. A ultrassonografia não é adequada como indicador de potencial reprodutivo.

\section{AGRADECIMENTOS}

À FAPESP pelo apoio financeiro em forma de bolsa de mestrado; aos criadores Flávio Pereira Aranha e Adriana Salles Zancaner Aranha (Fazenda da Bela Alvorada), por cederem os animais e acreditarem nesta pesquisa; ao estagiário Alexandre José Coutinho e aos funcionários da Fazenda da Bela Alvorada, pela grande ajuda nos dias de trabalho.

\section{REFERÊNCIAS}

ABDEL-RAZEK, A.; ALI, A. Development changes of bull (Bos taurus) genitalia as evaluated by caliper and ultrasonography. Reprod. Dom. Anim., v.40, p.23-27, 2005.

ARAVINDAKSHAN, J.P.; HONARAMOOZ, A.; BARTLEWSKI, P.M. et al. Pattern of gonadotropin secretion and ultrasonographic evaluation of developmental changes in the testis of early and late maturing bull calves. Theriogenology, v.54, p.339-354, 2000.

BRITO, L.F.C.; SILVA, A.E.D.F.; RODRIGUES, L.H. et al. Effect of age and genetic group on characteristics of the scrotum, tests and vascular cones, and on sperm production and semen quality in AI bulls in Brazil. Theriogenology, v.58, p.1175-1186, 2002.

BRITO, L.F.C.; SILVA, A.E.D.F.; UNANIAM, M.M. et al. Sexual development in early and late maturing Bos indicus and Bos indicus $x$ Bos Taurus crossbred bulls in Brazil. Theriogenology, v.62, p.1198-1217, 2004.

CARDILLI, D.J.; TONIOLLO, G.H.; PASTORE, A.A. et al. Alterações do padrão ultrassonográfico do parênquima testicular de bovinos jovens da raça Nelore. Acta Sci. Vet., v.37, p.367-370, 2009.

CHANDOLIA, R.K.; HONARAMOOZ, A.; OMEKE, B.C. et al. Assessment of development of the testes and acessory glands by ultrasonography in bull calves and associated endocrine changes. Theriogenology, v.48, p.119132, 1997.

DROST, W.T. Basic ultrasound physics. In: THRALL, D.E. Textbook of veterinary diagnostic radiology. 4.ed. Philadelphia: W. B. Sauders Company, 2002, p.20-27.

HAMM, B.; FOBBE, F. Maturation of the Testis: ultrasound evaluation. Ultrasound Med. Biol., v.21, p.143-147, 1995.

HORSTMAN, W.G.; HALUSZKA, M.M.; BURKHARD, T.K. Management of testicular masses incidentally discovered by ultrasound. $J$. Urology, v.151, p.1263, 1994. 
KASTELIC, J.P.; COOK, R.B.; PIERSON, R.A. et al. Relationships among scrotal and testicular characteristics, sperm production, and seminal quality in 129 beef bulls. Can. J. Vet. Res., v.65, p.111-115, 2001.

KRAUSE, D. Sistema reprodutor masculino. In: DIRKSEN, G.; GRÜNDER, U.; STÖBER, M. Rosenberger: Exame Clínico dos Bovinos. Rio de Janeiro: Guanabara Koogan, 1993, p.242-268.

PECHMAN, R.D.; EILTS, B.E. B-mode ultrasonography of the bull testicle. Theriogenology, v.27, p.431-441, 1987.

SILVA, A.; KASTELIC, J.P.; UNANIAM, M.M. et al. Ultrassonografia de machos Nelore na fase peripuberal. Rev. Bras. Reprod. Anim., v.21, p.34-36, 1997.
SIMON, S.D.; LEE, R.D.; MULHALL， J.P. Should all infertile males undergo urologic evaluation before assisted reproductive technologies? Two cases of testicular cancer presenting with infertility. Fertil. Steril., Birmingham, v.75, p.1226, 2001.

SISSON, S. Aparelho urogenital. In: GETTY, R. Anatomia dos animais domésticos. Rio de Janeiro: Guanabara Koogan, 1986. Cap. 42, p.881-886.

UNANIAM, M.M.; SILVA, A.; MACMANUS, C. et al. Características biométricas testiculares para avaliação de touros zebuínos da raça Nelore. Rev. Bras. Zootec., v.29, p.136-144, 2000. 